\title{
Calidad de vida relacionada con la salud de pacientes con tuberculosis pulmonar
}

\author{
Health-related quality of life in patients \\ with pulmonary tuberculosis
}

\author{
Diego Ivar Álvarez-López, ${ }^{*}$ Jocelyn Andrea Almada-Balderrama, ${ }^{*}$ \\ Mónica Paola Espinoza-Molina,* Gerardo Álvarez-Hernández*
}

*Universidad de Sonora, Hermosillo, México.

RESUMEN. Introducción: La tuberculosis pulmonar es una enfermedad crónica infecciosa que deteriora la calidad de vida relacionada con la salud (CVRS) de los personas que la padecen. El estado de Sonora ocupa el segundo lugar nacional en morbilidad y tercero en mortalidad por tuberculosis pulmonar, pero al momento no se ha evaluado a nivel local cómo se afecta la CVRS en los pacientes con tuberculosis pulmonar. Objetivo: Evaluar longitudinalmente la CVRS en adultos $\geq 18$ años de edad con tuberculosis pulmonar confirmada, que fueran atendidos en centros de salud de Hermosillo, Sonora. Métodos: Estudio transversal con 46 sujetos a los que se aplicó el cuestionario validado Short Form 36 (SF-36v2) para examinar su CVRS. Los resultados fueron comparados con las variables de género, fase de tratamiento (intensiva/sostén) y comorbilidades (presente/ ausente); adicionalmente, se describieron aspectos sociodemográficos como ocupación, educación e ingreso familiar. Resultados: La CVRS fue significativamente menor en mujeres, pacientes en fase intensiva y en los que tenían comorbilidades. El deterioro más notorio ocurrió en aspectos físicos y la percepción de salud global. Conclusión: Los sujetos con tuberculosis pulmonar perciben deterioro en su CVRS, pero el tratamiento farmacológico ejerce un efecto positivo en su bienestar.

Palabras clave: Calidad de vida relacionada a la salud, tuberculosis, México.

Abreviaturas:

CVRS $=$ Calidad de vida relacionada con la salud.

Mtb $=$ Mycobacterium tuberculosis.

SF-36v2 = Cuestionario validado Short Form 36.

TAES $=$ Tratamiento acortado estrictamente supervisado.

$\mathrm{TB}=$ Tuberculosis.

$\mathrm{TBP}=$ Tuberculosis pulmonar.

Correspondencia:

Dr. Gerardo Álvarez-Hernández

Universidad de Sonora, Hermosillo, México.

Correo electrónico: gerardo.alvarez@unison.mx;

galvarezh63@gmail.com

Trabajo recibido: 23-X-2019; aceptado: 27-XI-2019.
ABSTRACT. Introduction: Pulmonary tuberculosis (TBP) is a chronic infectious disease that impairs the health-related quality of life (HRQOL) of the patients who suffer from it. Sonora ranks second in national morbidity and third in mortality from TBP, but to our knowledge it has not been evaluated how HRQOL affects patients with TBP at the local level. Objective: To evaluate HRQOL in adults $\geq 18$ years of age with confirmed TBP seen at health centers in Hermosillo, Sonora. Methods: We carried out a cross-sectional study with 46 subjects, to whom the validated Short Form 36 questionnaire (SF-36v2) was applied to examine their HRQOL. The outcomes were compared according to the variables gender, phase of treatment phase (intensive/support) and comorbidities (present/absent); sociodemographic aspects such as occupation, education and family income were also described. Results: HRQOL was significantly lower in women, patients in intensive phase and those who had comorbidities. The most notorious deterioration occurred in physical aspects and the perception of global health. Conclusion: Subjects with TBP perceived deterioration in their HRQOL, but pharmacological treatment exerts a positive effect on their well-being.

Keywords: Health-related quality of life, tuberculosis, Mexico.

\section{INTRODUCCIÓN}

La tuberculosis (TB) es una enfermedad bacteriana de importancia mundial. Esta enfermedad es causada por Mycobacterium tuberculosis (Mtb) y representa un problema de salud pública global, pues cada año ocurren poco más de 10 millones de casos nuevos y 1.5 millones de muertes alrededor del mundo. Se estima, además, que $87 \%$ de la incidencia de la enfermedad ocurre en 30 países, ubicados principalmente en Asia y África. ${ }^{1}$ En México, entre 2006 y 2015 ocurrieron en promedio casi 20 mil casos nuevos por año de todas las formas de TB. ${ }^{2}$ En el estado de Sonora, al noroeste del país, la TB provoca alrededor de mil casos nuevos y cien muertes cada año; en tanto, Hermosillo, la capital del estado, concentra un tercio de la incidencia y la mitad de las muertes por esta enfermedad. ${ }^{2}$ 
El principal mecanismo de transmisión de la TB es por diseminación aérea; Mtb afecta principalmente a los pulmones, causándoles una inflamación granulomatosa necrotizante. ${ }^{3}$ Sin embargo, es común encontrar una diseminación multisistémica en personas enfermas, lo que deteriora su bienestar, particularmente, la calidad de vida relacionada con la salud (CVRS). ${ }^{4}$ Éste es un concepto multidimensional, que examina componentes físicos, cognitivos, emocionales y sociales de un individuo, y los relaciona con sus condiciones de salud o tratamiento. ${ }^{5-7}$

La medición de la CVRS ha mostrado ser útil para predecir los resultados de un tratamiento y los cambios en las condiciones de salud de los pacientes; ${ }^{8}$ asimismo, permite evaluar la percepción de los pacientes con enfermedades crónicas, transmisibles y no transmisibles, las cuales merman su bienestar físico, emocional y social. ${ }^{5,9,10}$

Uno de los instrumentos de medición más empleados para examinar la CVRS es el cuestionario validado Short Form 36 (SF-36v2). ${ }^{11}$ Este cuestionario consta de 36 preguntas con respuesta cerrada tipo Likert y explora ocho dimensiones del estado de salud: función física, función social, rol físico, rol emocional, salud mental, vitalidad, dolor corporal y percepción de la salud general. De esta manera, el SF-36v2 evalúa estados de salud individuales, tanto positivos como negativos.

En general, se acepta que la TB deteriora la CVRS de un paciente de forma global, aunque diversos estudios han documentado que los roles físicos y emocionales son los más afectados al comienzo del tratamiento, y mejoran una vez que lo finalizan. ${ }^{8,12-14}$ No obstante, en México, es escasa la evidencia al respecto, por lo que este estudio tiene como objetivo examinar las repercusiones físicas, psicológicas y sociales que la TB tiene en el bienestar de pacientes atendidos en unidades públicas de primer nivel de atención del estado de Sonora, el cual es considerado como de alta prioridad a nivel nacional debido a su carga de morbilidad y mortalidad. ${ }^{2}$

\section{MATERIAL Y MÉTODOS}

Mediante un estudio transversal, se evaluó el efecto de la TB sobre la CVRS en pacientes que recibieron atención médica en unidades de salud de primer nivel de atención en Hermosillo, Sonora, entre el primero de marzo y el 15 de junio de 2018. Para la selección de la muestra, se utilizó un diseño no probabilístico que incluyó a 46 sujetos convenientemente elegidos.

Los pacientes debían cumplir los siguientes criterios de inclusión: ser residentes de Sonora por, al menos, 12 meses previos a su inclusión, hablantes de español, de edad $>18$ años y que no presentaran limitaciones que afectaran su capacidad de contestar las preguntas aplicadas. El diagnóstico de TB pulmonar (TBP) se debió confirmar mediante baci- loscopia y estar bajo tratamiento acortado estrictamente supervisado (TAES) al momento del estudio, lo que debió ser evidenciado en la Plataforma Única de Información del Sistema Nacional de Vigilancia Epidemiológica, Módulo Tuberculosis (Secretaría de Salud, 2017). Fueron excluidos aquellos sujetos con TB extrapulmonar, cáncer pulmonar concomitante y aquéllos que no concluyeron el cuestionario. Todos los procedimientos del estudio recibieron la aprobación del Comité de Bioética en Investigación del Departamento de Medicina y Ciencias de la Salud de la Universidad de Sonora.

Para examinar la CVRS, se utilizó el cuestionario validado Short Form 36 (SF-36v2), el cual consta de 36 preguntas para evaluar ocho escalas: salud global, funcionamiento físico, rol físico, dolor corporal, rol emocional, vitalidad, salud mental y función social. El análisis de los datos se basó en el enfoque Rand. ${ }^{11}$ En breve, el sistema utiliza una escala Likert para asignar valores entre 0 (mínimo) y 100 (máximo) a cada ítem. Cada una de las ocho escalas agrupa un número diferente de ítems (entre dos y 10) para las que se obtiene el valor promedio, que es entonces considerado como la puntuación global de cada escala. Las puntuaciones de las ocho escalas del cuestionario están ordenadas de forma que, a mayor valor, mejor la calidad de vida del paciente.

Adicionalmente, se aplicó un cuestionario de 15 ítems para explorar aspectos sociodemográficos, como sexo, ocupación, características de la vivienda, ingreso familiar, escolaridad, así como la presencia de comorbilidades al momento de la encuesta.

Para examinar diferencias en la CVRS, se comparó a los sujetos de estudio de acuerdo con su sexo y la fase de tratamiento antifímico en que se encontraban, en la que se consideró una fase intensiva (hasta dos meses) y una fase de sostén (de tres a seis meses). Además, el puntaje obtenido en cada una de las escalas fue contrastado entre el grupo de pacientes con comorbilidades y el que no presentaba alguna enfermedad adicional que fuera autorreferida por los sujetos.

Para el análisis de datos, se empleó estadística descriptiva. Las variables se presentaron en tablas de frecuencia (media aritmética y error estándar). Las diferencias entre las variables de ambos grupos fueron examinadas mediante t de Student en las variables continuas y $\chi^{2}$ para las categóricas. Se probaron diferencias con hipótesis a dos colas, y valores de $p \leq a$ 0.05 fueron considerados estadísticamente significativas. El programa estadístico empleado fue el NCSS versión 12.0.

\section{RESULTADOS}

Un total de 46 sujetos fueron incluidos en el estudio; de los cuales, 13 (28\%) se encontraban en la fase intensiva y $33(72 \%)$ en la fase de sostén del tratamiento contra TB. De los sujetos de estudio, 37 (80\%) eran hombres. No se observaron diferencias significativas $(p=0.12)$ en la edad 
media de los sujetos que se encontraban en fase intensiva (47.17 \pm 14.53$)$ en comparación con los de la fase de sostén (39.37 \pm 15.07 ), cuyo un rango de edad era de 18-79 años. Al comparar las fases de tratamiento, no se observaron diferencias en la distribución por género $(p=0.25)$ ni en otras variables sociodemográficas estudiadas. Dentro de la muestra, sólo un sujeto declaró no contar con escolaridad alguna, 14 individuos (30\%) se encontraban sin ocupación asalariada y 35 (76\%) se encontraba sin pareja. Los detalles se despliegan en la Tabla 1.

Cuando comparamos la CVRS entre hombres y mujeres, se observaron diferencias estadísticamente significativas en todas las escalas examinadas, a excepción de la percepción de la «Salud global» $(p=0.075)$ y «Limitación de roles debido a problemas físicos» ( $p=0.899$ ). En general, los hombres presentaron un mayor puntaje que las mujeres en todas las escalas, excepto en la del dolor, en la que los varones tuvieron menor puntuación $(75.821 \pm 3.616)$ que las mujeres (80.012 \pm 5.256$)$, cuya diferencia fue significativa

Tabla 1: Características sociodemográficas de los sujetos de estudio de acuerdo con su fase de tratamiento.

\begin{tabular}{|c|c|c|c|}
\hline Variable & $\begin{array}{l}\text { Fase intensiva } \\
(n=13)\end{array}$ & $\begin{array}{l}\text { Fase de sostén } \\
\qquad(n=33)\end{array}$ & $p^{*}$ \\
\hline $\begin{array}{l}\text { Género } \\
\text { Masculino } \\
\text { Femenino }\end{array}$ & $\begin{array}{l}9(69.2) \\
4(30.8)\end{array}$ & $\begin{array}{r}28(84.9) \\
5(15.2)\end{array}$ & 0.2462 \\
\hline Edad (años) ${ }^{\star \star}$ & $47.17 \pm 14.53$ & $39.37 \pm 15.07$ & 0.1175 \\
\hline $\begin{array}{l}\text { Escolaridad } \\
\text { Con algún grado } \\
\text { de educación } \\
\text { básica }^{\star \star \star} \\
\text { Preparatoria y más }\end{array}$ & $\begin{array}{l}11(84.6) \\
1(7.7)\end{array}$ & $\begin{array}{l}23(69.7) \\
10(30.3)\end{array}$ & 0.1294 \\
\hline $\begin{array}{l}\text { Ocupación (sector) } \\
\text { Primario } \\
\text { Secundario } \\
\text { Terciario } \\
\text { Sin ocupación } \\
\text { asalariada }\end{array}$ & $\begin{array}{l}3(23.1) \\
4(30.8) \\
4(30.8) \\
2(15.4)\end{array}$ & $\begin{array}{r}4(12.1) \\
9(27.3) \\
8(24.2) \\
12(36.4)\end{array}$ & 0.4891 \\
\hline $\begin{array}{l}\text { Ingreso familiar } \\
\text { Sin ingreso } \\
\text { Hasta dos salarios } \\
\text { mínimos } \\
\text { Dos o más salarios } \\
\text { mínimos }\end{array}$ & $\begin{array}{l}3(23.1) \\
3(23.1) \\
7(53.9)\end{array}$ & $\begin{array}{l}5(15.2) \\
7(21.2) \\
21(63.6)\end{array}$ & 0.8119 \\
\hline $\begin{array}{l}\text { Estado civil } \\
\text { Vive en pareja } \\
\text { Vive sin pareja }\end{array}$ & $\begin{array}{l}4(30.8) \\
9(69.2)\end{array}$ & $\begin{array}{r}7(21.2) \\
26(78.8)\end{array}$ & 0.7024 \\
\hline
\end{tabular}

* Basado en una prueba exacta de Fisher.

** Basado en una prueba de t de Student (dos colas).

${ }^{* * \star}$ Educación básica incluye escolaridad primaria y secundaria. $(p=0.006)$. También se apreció que el funcionamiento físico de los varones tuvo la mayor diferencia (25.710) respecto de las mujeres, seguido por la limitación de roles debido a problemas emocionales (23.566), tal como se aprecia en la Tabla 2.

Al comparar la CVRS de los sujetos de acuerdo con la fase de tratamiento en la que se encontraban, se observaron diferencias estadísticamente significativas en los puntajes de todas las escalas examinadas; en general, los pacientes en fase de sostén obtuvieron puntajes más altos, tanto en los componentes de salud física como en los de mental. En ambas fases de tratamiento, la escala «Limitación de roles debido a problemas físicos» fue la de menor puntaje; en la intensiva fue de $39.461 \pm 8.707$, mientras que en la de sostén fue de $56.679 \pm 5.909$. En la fase intensiva, el puntaje más alto $(65.585 \pm 4.526)$ se apreció en la escala «Bienestar emocional»; en tanto en la fase de sostén, el puntaje más elevado (89.398 \pm 4.387$)$ ocurrió en el «Funcionamiento social». Además, se encontró que en las dimensiones «Funcionamiento social» (39.732) y «Limitación de roles debido a problemas emocionales» (32.108) hubo la mayor diferencia en la puntuación media entre los grupos; en este sentido, en todas las dimensiones se apreció un mayor puntaje en los sujetos que se encontraban en la fase de sostén (Tabla 3).

Cabe señalar que 20 (43\%) de los sujetos de estudio manifestaron presentar al menos una comorbilidad, siendo diabetes $(8 ; 17 \%), \mathrm{VIH}(4 ; 9 \%)$, hepatitis C $(3 ; 7 \%)$ y alcoholismo $(2 ; 4 \%)$ como las reportadas con mayor frecuencia. Además, dos (4\%) individuos expresaron tener algún grado de depresión. Al comparar el puntaje promedio de la CVRS entre los pacientes que reportaron alguna comorbilidad y los que no, se observó que estos últimos tuvieron puntajes más altos en todas las escalas, y las diferencias fueron estadísticamente significativas, a excepción de la dimensión «Salud global» $(p=0.091)$; la mayor diferencia en la puntuación media entre los grupos se encontró en la escala de «Funcionamiento físico» (23.257) y en el «Dolor» (21.283). La escala referente al dolor (86.376 \pm 2.994$)$ mostró el puntaje más alto en los sujetos sin comorbilidad; en los del grupo con comorbilidades, el bienestar emocional (70.950 \pm 4.220$)$ registró el mayor puntaje (Tabla 4).

\section{DISCUSIÓN}

Los hallazgos del presente estudio documentan diferencias en CVRS de pacientes con TB, que son residentes de una región de endemia media; tales diferencias se observaron entre hombres y mujeres, la fase de tratamiento antifímico y la presencia de comorbilidades. En general, se encontró una menor CVRS en las mujeres, en los pacientes en la fase intensiva de tratamiento y en los que tenían alguna 
Tabla 2: Comparación de las dimensiones de la CVRS, según el sexo.

\begin{tabular}{|c|c|c|c|c|c|}
\hline Dimensiones & $\begin{array}{l}\text { Femenino } \\
(n=9)\end{array}$ & $\begin{array}{l}\text { Masculino } \\
(n=37)\end{array}$ & Efecto* & Total & $p^{\star \star}$ \\
\hline Salud global & $51.752 \pm 7.077$ & $54.477 \pm 2.942$ & 2.725 & $53.933 \pm 2.704$ & 0.075 \\
\hline Limitación de roles debido a problemas físicos & $50.991 \pm 10.300$ & $51.309 \pm 5.610$ & 0.318 & $51.256 \pm 4.938$ & 0.899 \\
\hline Limitación de roles debido a problemas emocionales & $56.080 \pm 10.310$ & $79.646 \pm 6.579$ & 23.566 & $73.452 \pm 5.626$ & $<0.001$ \\
\hline Energía/fatiga & $52.520 \pm 5.977$ & $68.536 \pm 3.289$ & 16.016 & $65.058 \pm 3.017$ & $<0.001$ \\
\hline Dolor & $80.012 \pm 5.256$ & $75.821 \pm 3.616$ & 4.191 & $76.623 \pm 3.062$ & 0.006 \\
\hline Comparación respecto a 1 año previo & $48.949 \pm 11.024$ & $61.142 \pm 4.294$ & 12.193 & $59.561 \pm 4.154$ & $<0.001$ \\
\hline
\end{tabular}

CVRS = calidad de vida relacionada con la salud.

* Diferencia del puntaje promedio de los grupos de estudio.

${ }^{* \star}$ Basado en una prueba T de Student.

Tabla 3: Comparación de las dimensiones de la CVRS, según fase de tratamiento.

\begin{tabular}{|l|c|c|c|c|c|}
\hline \multicolumn{1}{|c|}{ Dimensiones } & $\begin{array}{c}\text { Intensivista } \\
(\mathbf{n = 1 3 )}\end{array}$ & $\begin{array}{c}\text { Sostén } \\
(\mathbf{n}=\mathbf{3 7})\end{array}$ & Efecto* $^{*}$ & Total $^{*}$ \\
\hline Salud global & $44.639 \pm 4.297$ & $58.104 \pm 3.128$ & 13.465 & $53.933 \pm 2.704$ & $<0.001$ \\
Funcionamiento físico & $56.448 \pm 6.343$ & $78.439 \pm 3.809$ & 21.991 & $71.475 \pm 3.488$ & $<0.001$ \\
Limitación de roles debido a problemas físicos & $39.461 \pm 8.707$ & $56.679 \pm 5.909$ & 17.218 & $51.256 \pm 4.938$ & $<0.001$ \\
Limitación de roles debido a problemas emocionales & $51.122 \pm 9.537$ & $83.230 \pm 6.237$ & 32.108 & $73.452 \pm 5.626$ & $<0.001$ \\
Energía/fatiga & $54.607 \pm 5.732$ & $69.705 \pm 3.338$ & 15.098 & $65.058 \pm 3.017$ & $<0.001$ \\
Bienestar emocional & $65.585 \pm 4.526$ & $79.584 \pm 7.742$ & 13.999 & $75.349 \pm 2.510$ & $<0.001$ \\
Funcionamiento Social & $49.666 \pm 9.016$ & $89.398 \pm 4.387$ & 39.732 & $70.501 \pm 4.268$ & $<0.001$ \\
Dolor & $62.858 \pm 6.587$ & $82.840 \pm 3.095$ & 19.982 & $76.623 \pm 3.062$ & $<0.001$ \\
Comparación respecto a 1 año previo & $50.253 \pm 6.426$ & $63.817 \pm 5.135$ & 13.564 & $59.561 \pm 4.154$ & $<0.001$ \\
\hline
\end{tabular}

CVRS $=$ calidad de vida relacionada con la salud.

* Diferencia del puntaje promedio de los grupos de estudio.

** Basado en una prueba $t$ de Student.

comorbilidad. Nuestros resultados son consistentes con estudios previos. ${ }^{15-18}$

En ese deterioro de la CVRS, las dimensiones más afectadas correspondieron a la limitación de roles debido a problemas físicos y a la percepción de salud global, lo cual es consistente con un estudio mexicano realizado previamente. ${ }^{19}$ Esto mismo fue observado en Pakistán, en donde dos de las tres dimensiones con puntajes más bajos correspondieron a las ya mencionadas. ${ }^{20}$

Aunque en la región la incidencia de TB es mayor en hombres, las mujeres percibieron su CVRS con un mayor deterioro que los hombres, lo que ya ha sido descrito en diversos estudios. Por ejemplo, Chang et al. ${ }^{20}$ encontraron en él que las mujeres con TB son más susceptibles de una menor CVRS. Dicho menoscabo puede ser explicado por distintas razones. En primer lugar, el temor al estigma social relacionado con la TB puede llevar a la mujer a negar su sintomatología y, por ende, buscar atención médica cuando su cuadro clínico es más severo. ${ }^{21}$ En segundo lugar, ciertos patrones sociales en algunas culturas, como la nuestra, in- crementan la vulnerabilidad de las mujeres, pues, a pesar de recibir el diagnóstico de TB, deben continuar con las labores domésticas asignadas por su familia, lo que puede significar una carga adicional en la vida de la paciente. ${ }^{22}$ En ese sentido, la estructura social de países en vías de desarrollo contribuye a que las mujeres tengan una mayor carga laboral y, por lo tanto, el impacto de la TB sobre ellas se extiende a sus familias y al desarrollo de la sociedad. ${ }^{23}$ En nuestro estudio, las subescalas de CVRS que presentaron una mayor diferencia entre hombres y mujeres fueron el funcionamiento físico y la limitación de roles debido a problemas emocionales, lo cual sugiere que la enfermedad podría ocasionar un significativo impacto psicosomático en las mujeres con TB.

Por otra parte, fue mejor percibida la CVRS de los sujetos que se encontraban en la fase de sostén de su tratamiento antifímico. Este hallazgo es similar al reportado en Uganda, en donde evaluaron la CVRS de 210 pacientes en tres diferentes etapas del manejo antifímico (antes de recibir tratamiento, fase intensiva y fase de sos- 
tén), encontrando una mejor CVRS en pacientes en fase de sostén. ${ }^{16}$ También estudios prospectivos han mostrado que los pacientes muestran una mejoría considerable en su CVRS conforme avanzan en su tratamiento. ${ }^{8,17,24}$ Específicamente, en nuestro estudio, la mayor diferencia en la CVRS entre ambas fases se apreció en las dimensiones de funcionamiento social y limitación de roles, debido a problemas emocionales que, a su vez, fueron dos de los tres puntajes más bajos registrados en pacientes en fase intensiva. Dicho resultado sugiere que además de la importancia que tiene el tratamiento para la curación médica de la enfermedad, también confiere un beneficio en el ámbito socioemocional del paciente.

Un aspecto que merece atención especial en la TB es su asociación con enfermedades transmisibles (por ejemplo, $\mathrm{VIH}$ ) y no transmisibles (por ejemplo, diabetes mellitus), y la potencial complejidad que representa su atención, así como el posible deterioro del bienestar de los pacientes. En nuestro estudio, apreciamos que los sujetos con alguna comorbilidad tuvieron un menor puntaje en todas las subescalas de la CVRS en comparación de los sujetos sin comorbilidades, siendo las más bajas aquellas referentes a la función física y al dolor físico. Estos resultados son consistentes con los reportados en estudios previos. ${ }^{25-27}$ Al respecto, es de especial importancia la diabetes y el $\mathrm{VIH}$, puesto que ambas enfermedades constituyen factores de riesgo para adquirir o reactivar la enfermedad. Un estudio en Irán que evaluó la CVRS en pacientes con diabetes y TB mostró puntajes inferiores a la media en todas las dimensiones de salud, especialmente en la salud física. ${ }^{25}$ Resultados similares se encontraron en un estudio prospectivo realizado en India, donde la CVRS fue peor en individuos con diabetes y TB, especialmente en los sujetos con glucemia no controlada, en comparación con sujetos sin diabetes..$^{28}$ Un estudio prospectivo en México encontró que pacientes con diabetes y TB tienen mani- festaciones clínicas más graves, retraso en negativización de baciloscopia y una mayor probabilidad de fracaso del tratamiento, recurrencia y recaída, lo que podría afectar negativamente la CVRS de los pacientes. ${ }^{29}$

Adicionalmente, existe evidencia de que pacientes con el binomio TB/VIH presentan una peor CVRS que sujetos sin su presencia. Por ejemplo, en Etiopía se realizó un estudio en pacientes con TB y coinfección por $\mathrm{VIH}$, donde la CVRS fue peor en todas las escalas que en pacientes que sólo presentaban $\mathrm{VIH} .{ }^{26}$ En otra investigación realizada en Sudáfrica, la CVRS en pacientes con TB/VIH fue menor que en pacientes $\sin \mathrm{VIH}$, particularmente en las subescalas de función física, dolor y salud global, lo cual es consistente con nuestros resultados. ${ }^{27}$

Así, nuestros hallazgos indican que tener una o más enfermedades agregadas a la TB tiene un impacto agravante en la CVRS del paciente. No podemos afirmar, sin embargo, si un tratamiento prolongado o diferente en pacientes con TB y comorbilidades tendría un efecto beneficioso en la CVRS, por lo cual es recomendable que nuevos estudios se lleven a cabo abordando este aspecto en la región. $Y$ es que se ha señalado que resultados menores en las subescalas de salud mental y emocional en pacientes con comorbilidades estarían asociadas con la estigmatización de la TB y la coinfección por otros agentes como VIH, lo que lleva a aislamiento social. ${ }^{30}$

Nuestro estudio tiene algunas limitaciones metodológicas que deben ser tomadas en cuenta. Dado que se trata de un estudio transversal, no es posible establecer relaciones causa-efecto. Además, el cuestionario que empleamos (SF-36v2), aunque de amplio uso internacional, no fue específicamente validado para la población en estudio, lo que provocaría potencial sesgo de información en los resultados observados. Aun así, nuestros hallazgos son similares con el otro y único estudio mexicano publicado respecto del tema. ${ }^{18}$

Tabla 4: Comparación de las dimensiones de la CVRS, según ausencia o presencia de comorbilidades.

\begin{tabular}{|l|c|c|r|r|r|}
\hline Dimensiones & $\begin{array}{c}\text { Con comorbilidades } \\
(\mathbf{n}=\mathbf{2 0})\end{array}$ & $\begin{array}{c}\text { Sin comorbilidades } \\
(\mathbf{n}=\mathbf{2 5})\end{array}$ & Efecto* & Total & $\mathbf{p}^{\star *}$ \\
\hline Salud global & $52.700 \pm 3.507$ & $54.706 \pm 4.136$ & 2.006 & $53.933 \pm 2.704$ & 0.091 \\
Funcionamiento físico & $59.265 \pm 5.781$ & $82.522 \pm 3.802$ & 23.257 & $71.475 \pm 3.488$ & $<0.000$ \\
Limitación de roles debido a problemas físicos & $44.606 \pm 7.129$ & $55.169 \pm 6.211$ & 10.563 & $51.256 \pm 4.938$ & $<0.001$ \\
Limitación de roles debido a problemas emocionales & $65.801 \pm 8.472$ & $79.968 \pm 7.272$ & 14.167 & $73.452 \pm 5.626$ & $<0.001$ \\
Energía/fatiga & $61.443 \pm 4.605$ & $68.561 \pm 4.082$ & 7.118 & $65.058 \pm 3.017$ & $<0.001$ \\
Bienestar emocional & $70.950 \pm 4.220$ & $78.857 \pm 3.109$ & 7.907 & $75.349 \pm 2.510$ & $<0.001$ \\
Funcionamiento Social & $67.668 \pm 6.516$ & $72.639 \pm 5.991$ & 4.971 & $70.501 \pm 4.268$ \\
Dolor & $65.093 \pm 5.147$ & $86.376 \pm 2.994$ & 21.283 & $76.623 \pm 3.062$ & $<0.001$ \\
Comparación respecto a 1 año previo & $54.543 \pm 6.663$ & $64.560 \pm 5.537$ & 10.017 & $59.561 \pm 4.154$ & $<0.001$ \\
\hline
\end{tabular}

CVRS = calidad de vida relacionada con la salud.

* Diferencia del puntaje promedio de los grupos de estudio.

${ }^{* *}$ Basado en una prueba t de Student. 


\section{CONCLUSIONES}

La TB es una enfermedad que tiene repercusiones negativas en la salud física y emocional del paciente. Lo que pudimos observar es que el régimen farmacológico logra mejoras sustanciales en ambos ámbitos del bienestar de un paciente. Debido a la aún elevada prevalencia nacional y regional de la TB, especialmente en poblaciones vulnerables, es conveniente que sistemáticamente se evalúe la CVRS en pacientes mexicanos con TB, lo que contribuiría a avanzar el conocimiento de la enfermedad, incorporando rutinariamente elementos psicosociales a los aspectos puramente médicos que son usados en la atención de los pacientes con TB. Este último aspecto podría incrementar el apego terapéutico y reducir el abandono del tratamiento.

\section{Agradecimientos}

Los autores del presente trabajo agradecen el apoyo del Departamento de Medicina y Ciencias de la Salud, así como del personal de epidemiología y enfermería que atienden a pacientes con tuberculosis en centros de salud de la ciudad de Hermosillo, Sonora, para que esta investigación pudiera efectuarse de una forma respetuosa y profesional.

\section{REFERENCIAS}

1. World Health Organization. Global Tuberculosis Report 2018. Geneva: World Health Organization; 2018 [fecha de consulta: 23 de octubre, 2019]. Accesible en: https://www.who.int/tb/publications/global_report/en/

2. Secretaría de Salud Sonora. Dirección General de Promoción a la Salud y Prevención de Enfermedades. Situación de la tuberculosis en Sonora, 2016 (del 25 al 31 de diciembre del 2016. Semana epidemiológica 52). México; 2017.

3. Dheda K, Barry CE 3rd, Maartens G. Tuberculosis. Lancet 2016;387(10024):1211-1226. https://doi.org/10.1016/s01406736(15)00151-8

4. Giraldo GN, Valencia AD, Cardona-Arias JA. Calidad de vida relacionada con la salud en tuberculosis: Revisión sistemática y metaanálisis. Infectio 2018;22(3):124-130.

5. Driscoll S, Gregory DM, Fardy JM, Twells LK. Long-term health-related quality of life in bariatric surgery patients: A systematic review and meta-analysis. Obesity (Silver Spring) 2016;24(1):60-70. https://doi. org/10.1002/oby.21322

6. Kent EE, Ambs A, Mitchell SA, Clauser SB, Smith AW, Hays RD. Health-related quality of life in older adult survivors of selected cancers: data from the SEER-MHOS linkage. Cancer 2015;121(5):758765. https://doi.org/10.1002/cncr.29119

7. Mohammed S, Nagla S, Morten S, Asma E, Arja A. IIIness perceptions and quality of life among tuberculosis patients in Gezira, Sudan. Afr Health Sci 2015;15(2):385-393. https://doi.org/10.4314/ ahs.v15i2.11

8. Jaber AA, Khan AH, Syed Sulaiman SA, Ahmad N, Anaam MS. Evaluation of health-related quality of life among tuberculosis patients in two cities in Yemen. PLoS One 2016;11(6):e0156258. https://doi. org/10.1371/journal.pone.0156258
9. Kragt L, Dhamo B, Wolvius EB, Ongkosuwito EM. The impact of malocclusions on oral health-related quality of life in children-a systematic review and meta-analysis. Clin Oral Investig 2016;20(8):1881-1894. https://doi.org/10.1007/s00784-015-1681-3

10. Van der Have M, Van der Aalst KS, Kaptein AA, et al. Determinants of health-related quality of life in Crohn's disease: a systematic review and meta-analysis. J Crohns Colitis 2014;8(2):93-106.https://doi. org/10.1016/j.crohns.2013.04.007

11. Ware JE Jr, Sherbourne CD. The MOS 36-item short-form health survey (SF-36) I. Conceptual framework and item selection. Med Care 1992;30:473-483.

12. Alene KA, Clements ACA, McBryde ES, et al. Mental health disorders, social stressors, and health-related quality of life in patients with multidrug-resistant tuberculosis: A systematic review and meta-analysis. J Infect 2018;77(5):357-367. https://doi. org/10.1016/j.jinf.2018.07.007

13. Ahmad N, Javaid A, Syed Sulaiman SA, et al. Effects of multidrug resistant tuberculosis treatment on patients' health related quality of life: Results from a Follow Up Study. PLoS One 2016;11(7):e0159560. https://doi.org/10.1371/journal.pone.0159560

14. Bauer M, Ahmed S, Benedetti A, et al. Health-related quality of life and tuberculosis: a longitudinal cohort study. Health Qual Life Outcomes 2015;13:65. https://doi.org/10.1186/s12955-015-0250-4

15. Monteiro S, Rodrigues V. A qualidade de vida da pessoa com tuberculose: em regime de toma observada. Acta Med Port 2011;24 Suppl 2:523-530.

16. Kisaka SMB, Rutebemberwa E, Kasasa S, Ocen F, Nankya-Mutyoba J. Does health-related quality of life among adults with pulmonary tuberculosis improve across the treatment period? A hospital-based cross sectional study in Mbale Region, Eastern Uganda. BMC Res Notes 2016;9:467. https://doi.org/10.1186/s13104-016-2277-y

17. Mamani M, Majzoobi MM, Ghahfarokhi SM, Esna-Ashari F, Keramat F. Assessment of health-related quality of life among patients with tuberculosis in Hamadan, Western Iran. Oman Med J 2014;29(2):102105. https://doi.org/10.5001/omj.2014.25

18. González-Baltazar R, Lupercio-Heredia A, Hidalgo-Santacruz G, León-Cortés SG, Salazar-Estrada JG. Calidad de vida relacionada con la salud y trabajo en pacientes con tuberculosis en Guadalajara, (México). Salud Uninorte 2013;29(1):13-21.

19. Malik M, Nasir R, Hussain A. Health related quality of life among TB patients: question mark on performance of $T B$ DOTS in Pakistan. J Trop Med 2018;2018:2538532. https://doi. org/10.1155/2018/2538532

20. Chang B, Wu AW, Hansel NN, Diette GB. Quality of life in tuberculosis: A review of the english language literature. Qual Life Res 2004;13(10):1633-1642. https://doi.org/10.1007/s11136-004-0374-1

21. Hudelson P. Gender differentials in tuberculosis: The role of socioeconomic and cultural factors. Tuber Lung Dis 1996;77(5): 391-400. https://doi.org/10.1016/s0962-8479(96)90110-0

22. Nair DM, George A, Chacko KT. Tuberculosis in Bombay: new insights from poor urban patients. Health Policy Plan 1997;12(1):77-85. https:// doi.org/10.1093/heapol/12.1.77

23. Diwan VK, Thorson A. Sex, gender and tuberculosis. Lancet 1999;353(9157):1000-1001. https://doi.org/10.1016/s01406736(99)01318-5

24. Marra CA, Marra F, Colley L, Moadebi S, Elwood RK, Fitzgerald JM. Health-related quality of life trajectories among adults with tuberculosis: differences between latent and active infection. Chest 2008;133(2):396-403. https://doi.org/10.1378/chest.07-1494 
25. Shahdadi H, Salarzaee M, Balouchi A. Quality of life of diabetic patients with smear positive PTB in southeastern Iran: A cross-sectional study in a poor region of Iran. Indian J Tuberc 2018;65(2):159-163. https://doi.org/10.1016/j.ijtb.2017.08.035

26. Deribew A, Tesfaye M, Hailmichael Y, et al. Tuberculosis and HIV co-infection: its impact on quality of life. Health Qual Life Outcomes 2009;7:105. https://doi.org/10.1186/1477-7525-7-105

27. Louw K, Peltzer K, Naidoo P, Matseke G, Mchunu G, Tutshana B. Quality of life among tuberculosis (TB), TB retreatment and/or TBHIV co-infected primary public health care patients in three districts in South Africa. Health Qual Life Outcomes 2012;10:77. https://doi. org/10.1186/1477-7525-10-77

28. Siddiqui AN, Khayyam KU, Siddiqui N, Sarin R, Sharma M. Diabetes prevalence and its impact on health-related quality of life in tuberculosis patients. Trop Med Int Health 2017;22(11):1394-1404. https://doi.org/10.1111/tmi.12968

29. Jiménez-Corona ME, Cruz-Hervert LP, García-García L, et al. Association of diabetes and tuberculosis: impact on treatment and post-treatment outcomes. Thorax 2013;68(3):214-220. https://doi. org/10.1136/thoraxjnl-2012-201756

30. Kastien-Hilka T, Abulfathi A, Rosenkranz B, Bennett B, Schwenkglenks $\mathrm{M}$, Sinanovic $\mathrm{E}$. Health-related quality of life and its association with medication adherence in active pulmonary tuberculosis- $A$ systematic review of global literature with focus on South Africa. Health Qual Life Outcomes 2016;14:42. https://doi.org/10.1186/s12955-016-0442-6

Conflicto de intereses: Los autores declaran no tener conflicto de intereses. 